\title{
Legionnaires' disease
}

\author{
Ingrid A. Evans ${ }^{\mathrm{A}}$, Andrew J. N. Marich ${ }^{\mathrm{B}}$ \\ and Peter Harding $\mathrm{C}$ \\ ${ }^{\mathrm{A}}$ NSW Public Health Officer Training Program, \\ NSW Department of Health \\ ${ }^{\mathrm{B} C o m m u n i c a b l e ~ D i s e a s e s ~ B r a n c h, ~ N S W ~ D e p a r t m e n t ~ o f ~ H e a l t h ~}$ \\ ${ }^{\mathrm{C}}$ Department of Health and Building, City of Sydney Council
}

\section{Epidemiology of Legionnaires' disease}

Legionnaires' disease was only recently identified in 1976 after an outbreak of pneumonia in people attending a convention of the American Legion in Philadelphia, United States of America. The causative agent was identified as a previously unknown bacterium, named Legionella. Legionnaires' disease is a form of bacterial pneumonia and is clinically difficult to diagnose. It usually starts with general malaise, lack of appetite, muscle aches and headache, followed by high fever, chills, a dry cough and pneumonia, with occasional abdominal pain and diarrhoea. The infection is treated with antibiotics.

Overall mortality from Legionnaires' disease is approximately $5 \%$, but is higher in hospitalised patients (up to $40 \%$ ). Mortality was previously thought to be around $10 \%$; however, newer diagnostic tests have revealed a wider spectrum of disease, with milder cases than were previously detected.

Despite the interest that surrounds outbreaks, most cases of Legionnaires' disease occur sporadically. The mode of transmission depends on the Legionella species, but disease is usually transmitted through inhalation of infectious particles from contaminated water (Legionella pneumophila) or soil (L. longbeachae). There has been no reported person-to-person transmission. The incubation period is $2-10$ days, usually 5-6 days. Cases are more common in the summer and autumn months. Risk factors for Legionnaires' disease include increasing age, smoking and being immunocompromised.

There are between 40 and 90 cases of Legionnaires'disease reported in New South Wales (NSW) every year, with an upward trend in national notifications observed over the last 15 years. It is unclear if this is due to a real increase in infections, or to increased publicity and case finding. In NSW, cases of $L$. pneumophila occur at a higher rate in urban areas, probably due to the higher density of cooling towers.

\section{Management of legionellosis}

There have been at least eight outbreaks or clusters of Legionnaires' disease in NSW in the last 15 years.
Identifying the source of sporadic or outbreak-related cases of Legionnaires' disease is always difficult because the causal organisms are common in the environment and test results usually follow some time after the exposure period, by which time the contamination has been resolved.

The most recent outbreak involved 10 cases who were exposed at Sydney's Circular Quay in January 2007.1 Public health staff and environmental health officers from the local public health unit worked in collaboration with NSW Department of Health and the City of Sydney Council to investigate the outbreak.

The City of Sydney Council maintains a register of almost 1400 water cooling systems within its area. The register is updated annually and contains such information as the type of system and the contact details of the owner of the premises and the maintenance company. Every cooling tower in the area is inspected at least once every three years, and more often if there is a history of high Legionella levels or if the cooling towers are situated in highly populated areas.

If a sampled cooling tower has Legionella levels of 10-1000 CFU/mL, warning letters are served on building owners recommending that they review their maintenance procedures in order to comply with the regulations, and a further sample of water is taken to follow up on recommended disinfection procedures. For Legionella levels of $1000 \mathrm{CFU} / \mathrm{mL}$ and over, a notice is served on the building owner to immediately shut down the system and carry out disinfection of the cooling tower, and a follow-up sample of water is taken for analysis. Shut-down notices are also served when a cooling tower has repeated levels of Legionella between 100 and $1000 \mathrm{CFU} / \mathrm{mL}$.

Future directions for the City of Sydney Council include: the adoption of the NSW Health endorsed Legionella Management Plan; implementation of a risk-based audit regime for cooling towers; notification of any failures or potential risks encountered to the local public health unit; and the implementation of education programs for building managers and maintenance companies.

\section{Reference}

1. Communicable Diseases Branch, NSW Department of Health Communicable Diseases Report, NSW, January and February 2007. N S W Public Health Bull 2007; 18(4): 66-71. doi:10.1071/NB07032 